Physics

Physics Research Publications

Scanning tunneling microscope study of striated carbon ridges in few-layer epitaxial graphene formed on $4 \mathrm{H}$-silicon carbide (0001)
S. E. Harrison
M. A. Capano

R. Reifenberger 


\title{
Scanning tunneling microscope study of striated carbon ridges in few-layer epitaxial graphene formed on $4 \mathrm{H}$-silicon carbide (0001)
}

\author{
S. E. Harrison, ${ }^{1, a)}$ M. A. Capano, ${ }^{1}$ and R. Reifenberger ${ }^{2}$ \\ ${ }^{1}$ School of Electrical and Computer Engineering, Birck Nanotechnology Center, Purdue University, \\ West Lafayette, Indiana 47907, USA \\ ${ }^{2}$ Department of Physics, Birck Nanotechnology Center, Purdue University, West Lafayette, \\ Indiana 47907, USA
}

(Received 31 October 2009; accepted 22 January 2010; published online 22 February 2010)

\begin{abstract}
Atomically resolved scanning tunneling microscope images of carbon ridge defects found in few-layer graphene formed on the $\mathrm{C}$-face $(000 \overline{1})$ of $4 \mathrm{H}$-silicon carbide reveal a striated exterior surface formed from out-of-plane distortions of the hexagonal graphene lattice. While ridge formation is likely explained by compressive in-plane stresses coupled with the small values of the bending modulus for few-layer graphene, the striated structure along the ridges argues for a localized unidirectional stress in the material directed along the ridge length. () 2010 American Institute of Physics. [doi:10.1063/1.3323092]
\end{abstract}

An understanding of how structural defects affect electron transport in few-layer graphene (FLG) is a critical step toward the development of high-performance carbon-based nanoelectronics. Defects in FLG can cause uncontrolled strain fields that produce spatial fluctuations in the electron density and the Dirac point and will serve to alter electron transport. ${ }^{1}$ A promising route to FLG synthesis is the sublimation of $\mathrm{Si}$ from the $\mathrm{Si}$-face $[\mathrm{SiC}(0001)]$ and $\mathrm{C}$-face $[\mathrm{SiC}(000 \overline{1})]$ of $4 \mathrm{H}$-silicon carbide $(4 \mathrm{H}-\mathrm{SiC})$ at temperatures in excess of $1300{ }^{\circ} \mathrm{C},{ }^{2-6}$ producing FLG with nonuniform thickness on an insulating substrate. ${ }^{7-10}$ Recent mobility measurements of graphitized $\mathrm{C}$-face $\mathrm{SiC}$ substrates find values near $18,100 \mathrm{~cm}^{2} / \mathrm{V} \mathrm{s}$ (Ref. 11) which are still well below the intrinsic limit found in suspended single-layer graphene sheets $\left(\sim 200,000 \mathrm{~cm}^{2} / \mathrm{V} \mathrm{s}\right) .^{12}$ Interestingly, electron mobilities reported on FLG grown on the C-face of SiC seem to be considerably higher than mobilities measured on the Si-face. ${ }^{13,14}$ While the origin of this difference is not yet clear, a distinguishing dissimilarity between $\mathrm{C}$-face and Siface FLG is the presence of a carbon ridge network that develops on the $\mathrm{C}$-face material.

At temperatures above $1475{ }^{\circ} \mathrm{C}$, two predominant features on graphitized $\mathrm{C}$-face $\mathrm{SiC}$ substrates have been reported: ${ }^{15}$ (i) the formation of interlocking ridge networks and (ii) long-range moiré superlattices with periodicities much greater than the $\mathrm{C}-\mathrm{C}$ spacing. The one-dimensional (1D) ridge networks form to relieve compressive stress while the observed moiré patterns result from a relative rotation between two graphene sheets. While atomically resolved scanning tunneling microscopy (STM) studies of the moiré superlattices have appeared, ${ }^{15,16}$ comparable STM studies to investigate the larger scale carbon ridges have not received comparable attention. To address this issue, three samples of FLG were prepared by annealing $\mathrm{SiC}$ at growth temperatures of 1475,1500 , and $1550{ }^{\circ} \mathrm{C} .{ }^{15,17,18}$ This study focuses on FLG grown on the $\mathrm{C}$-face of $\mathrm{SiC}$ because of the higher mobilities that have been reported. ${ }^{13,14}$ The features reported

${ }^{\text {a)} E l e c t r o n i c ~ m a i l: ~ s e h a r r i s @ p u r d u e . e d u . ~}$ below were consistently reproduced between the three samples. ${ }^{19,20}$

The 1D ridges, found on all samples of FLG grown for this study, formed interconnected networks that divided the atomically flat graphene overlayer into large tilelike regions. Ridge dimensions varied in height from $\sim 5$ to $30 \mathrm{~nm}$ with apparent widths ranging from $\sim 50$ to $450 \mathrm{~nm}$. Moiré superlattices on flat FLG were commonly found in close proximity to the ridges. Similar ridgelike networks have been reported in carbon nanolayers deposited on a $\mathrm{Ni}$ substrate from a dc discharge in a gaseous methane-hydrogen mixture. ${ }^{21}$

Figure 1(a) is an STM image that shows the exterior surface of a ridge has a striated morphology that is far rougher than the atomically flat graphene bordering the ridge. $^{22}$ Figure $1(\mathrm{~b})$ shows a spatial derivative of Fig. 1(a) that is useful to better visualize the ridge striations. A typical constant current scan on top of a ridge is given in Fig. 1(c). The striations observed exhibit nonuniform spacing in a direction perpendicular to the ridge orientation [see Fig. 1(d)]. The separation between adjacent striations ranged from $\sim 0.5$ to $30 \mathrm{~nm}$, and each striation varied in apparent height from $\sim 0.02$ to $7 \mathrm{~nm}$. No correlation between sample growth temperature and ridge striation morphology was observed. A general trend is that ridges seem less prevalent on the $1475{ }^{\circ} \mathrm{C}$ sample when compared with the 1500 and $1550{ }^{\circ} \mathrm{C}$ samples.

Figure 1(e) shows an atomic scale STM image from the top of a striated ridge that reveals a hexagonal periodicity of $0.243 \pm 0.021 \mathrm{~nm}$. Atomic scale images like Fig. 1(e) indicate that ridge striations are formed from out-of-plane distortions in the hexagonal graphene lattice, producing what appears to be buckling defects between adjacent rows of hexagons, similar to defects reported near step edges in highly oriented pyrolytic graphite. ${ }^{23}$ Figure $1(f)$ is a line profile along a striation. The measured periodicity is $0.26 \pm 0.01 \mathrm{~nm}$ and indicates the striation is aligned along $[11 \overline{2} 0]$. The subtended dihedral angle formed by the out of plane buckling forming the striation is estimated to be $\sim 160^{\circ}$. 


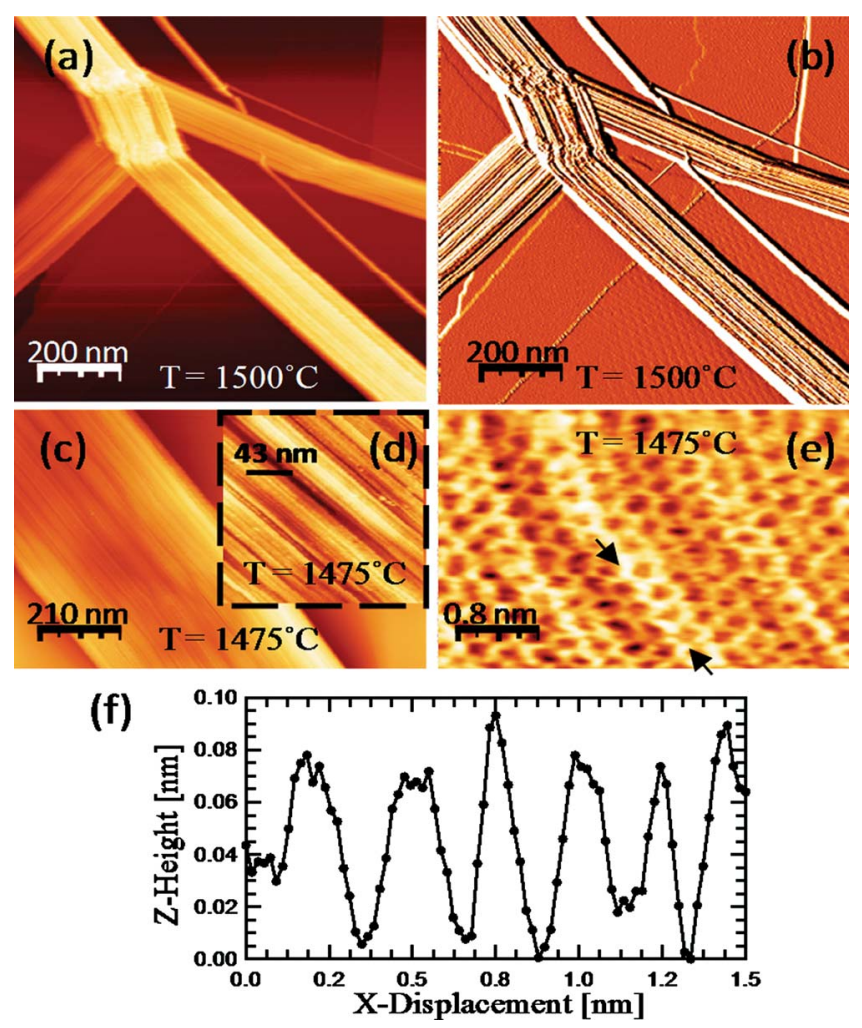

FIG. 1. (Color online) In (a), an STM image $\left(1.0 \times 1.0 \mu \mathrm{m}^{2}\right)$ of a network of interlocking striated ridges. Image (c) is the derivative of (b). In (c), a $1.1 \times 0.76 \mu \mathrm{m}^{2}$ image of an isolated striated ridge. In (d), an image (210 $\times 210 \mathrm{~nm}^{2}$ ) of ridge striations exhibiting nonuniform spacing. In (e), a $4.0 \times 2.7 \mathrm{~nm}^{2}$ atomically resolved image of ridge striations created by sharp bends in the graphene lattice. A line profile obtained across the ridge striation indicated by the black arrows in (e) is provided in (f). Scan parameters for (a) were $V_{\text {bias }}=0.3 \mathrm{~V}$ and $I_{\text {set }}=0.2 \mathrm{nA}$, (c) and (d) $V_{\text {bias }}=0.31 \mathrm{~V}$ and $I_{\text {set }}=0.2 \mathrm{nA}$, and (e) $V_{\text {bias }}=0.077 \mathrm{~V}$ and $I_{\text {set }}=5.6 \mathrm{nA}$.

Given the large variability in dimensions among ridges found on the same sample, the parallel striated exterior that is found on all ridges, regardless of sample growth temperature or ridge orientation to the crystal lattice, is remarkable and argues for a simple but universal origin. A membrane of thickness $t$ can either compress or bend under a compressive stress. If the membrane thickness is sufficiently small, bending eventually becomes the favored deformation since the bending modulus scales as $t^{3}$. One way to relax compressive stresses is to form a ridgelike bending deformation in which stress is condensed into narrow $1 \mathrm{D}$ ridges having no striations. $^{24}$

It is interesting to further consider the origin of the striations. The introduction of a physical height constraint is one way to understand how striations in a ridge might develop. ${ }^{25}$ Since there is no such height constraint in the growth of FLG, the origin of the striations requires additional factors and argues for a coupling between a bending deformation in the FLG (which produces a ridge) with a stretching deformation along the ridge (which produces striations). Such a scenario requires a longitudinal tensile stress directed along a ridge. Taken together, these two factors provide an explanation for the observed striations.

While the compressive strain produced during ridge formation is easy to visualize, the unidirectional strain along the ridge length is less intuitive. Prior work on the mechanics of membrane deformation has shown that parallel striations (also called wrinkles) form when a thin film, weakly coupled
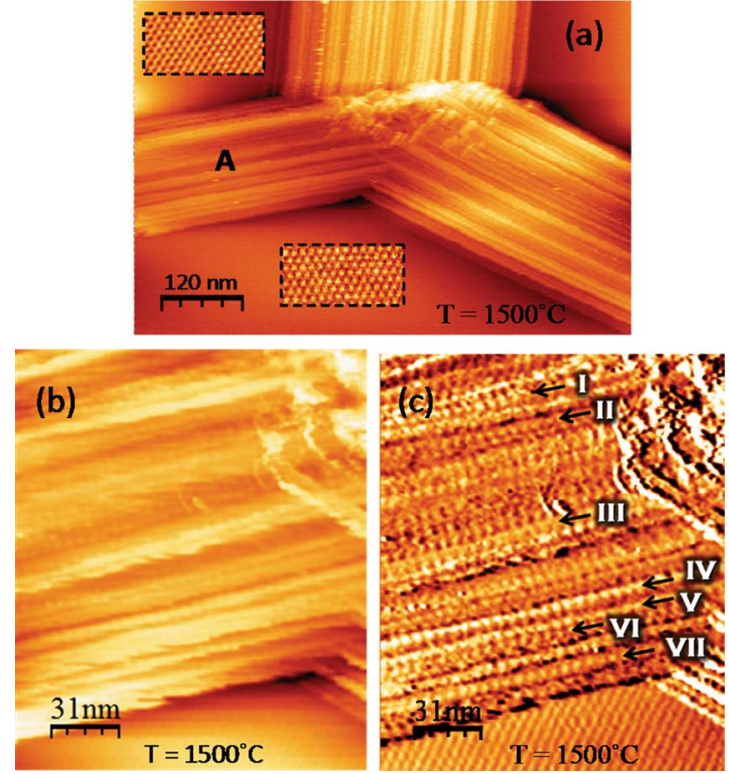

FIG. 2. (Color online) In (a), an STM image $\left(642 \times 388 \mathrm{~nm}^{2}\right)$ of a ridge network surrounded on either side by moiré superlattices with constant periodicities of $3.9 \mathrm{~nm}$ (see insets). In (b), a $165 \times 180 \mathrm{~nm}^{2}$ topographic image of ridge "A" showing $1 \mathrm{D}$ superlattices within the ridge striations. Image (c) is the derivative of (b). Scan parameters were $V_{\text {bias }}=0.15 \mathrm{~V}$ and $I_{\text {set }}$ $=0.5 \mathrm{nA}$.

to a substrate, is subjected to a tensile stress. The film relieves stress by forming a series of periodic wrinkles in the direction parallel to the applied stress. ${ }^{26,27}$ An accompanying longitudinal strain results due to fixed or clamped membrane boundaries that prevent the film from relaxing laterally. Scaling models have been developed to predict the spacing and amplitude of compressive wrinkles as well as their characteristic width. ${ }^{28-33}$ It is difficult at this time to apply these scaling models directly to the problem at hand, but it appears inescapable that FLG must be weakly bound to the underlying $\mathrm{C}$-face of $\mathrm{SiC}$ for striated ridges to develop.

Previous STM studies have reported 2D moiré superlattices in flat regions of FLG that lie in close proximity to ridges. ${ }^{15}$ Accurate measurements of the superlattice periodicity $\mathrm{P}$ can be used to infer the angular rotation $\Theta$ between two flat graphene layers according to $P=(d / 2) / \sin (\Theta / 2)$ where $d=0.246 \mathrm{~nm}$ is the lattice constant for a graphene lattice. $^{34-36}$

Interestingly, evidence for 1D superlattice features along the striations of a ridge was found in this study. The observed 1D superlattices are much larger than atomic periodicities, suggesting that they too are related to a periodic commensurability between $\mathrm{C}$-atoms in two adjacent layers.

Ridge "A" in Fig. 2(a) provides a typical example of 1D superlattice features. This ridge is surrounded on either side by well defined moiré superlattices (see insets) having a constant periodicity measuring $P=3.9 \mathrm{~nm}$, implying $\Theta=3.6^{\circ}$. Figure 2(b) shows an STM image from the topmost section of ridge A. In Fig. 2(c), a spatial derivative of Fig. 2(b) is provided to accentuate the 1D superlattice features aligned in straight parallel rows throughout the striated ridge. Measurements obtained from prominent rows in Fig. 2(c) provide estimates of the $1 \mathrm{D}$ periodicities $(P)$ as follows: I: $P=4.54 \mathrm{~nm}$; II: $P=5.00 \mathrm{~nm}$; III: $P=5.55 \mathrm{~nm}$; IV: $P$ $=5.55 \mathrm{~nm} ; \mathrm{V}: P=4.54 \mathrm{~nm} ; \mathrm{VI}: P=5.55 \mathrm{~nm}$; and VII: $P$ $=5.00 \mathrm{~nm}$. 
The measured periodicities $(4.5-5.6 \mathrm{~nm})$ from striations I-VII in Fig. 2(c) are larger than the periodicity found in the nearby flat graphene $(3.9 \mathrm{~nm})$. Assuming this periodicity is related to a rotation between two layers of graphene, this observation implies (i) a nonuniform rotation that rapidly varies across a ridge, and (ii) a consistently smaller rotation angle than measured in flat FLG. ${ }^{37}$ Estimates for $\Theta$, the rotational mismatch in the striations, vary between $2.5^{\circ}$ and $3.1^{\circ}$. Evidently, after a ridge forms, the nonuniform stress that develops can cause the topmost layers of graphene inside a ridge to relax in a highly nonuniform way.

The formation of striated ridges in FLG on the C-face of $\mathrm{SiC}$ serves to condense stress into a localized 1D network, leaving large areas of flat FLG that are relatively stress free. Furthermore, the observation of a distinct ridgelike network suggests that FLG is not tightly bound to the C-face of SiC. The absence of ridge formation on the thinner FLG grown on the Si-face ${ }^{8}$ implies it is more tightly bound to the Si-face of $\mathrm{SiC}$ than typical for the C-face. However, as the thickness of FLG on the Si-face increases, it is likely that a ridge network will appear. It also follows that thermal processing of mechanically exfoliated FLG, weakly bound to any supporting substrate, will likely develop a ridge network. The ubiquitous occurrence of these striated ridge defects may have far reaching consequences whenever FLG is thermally processed to fabricate nanoelectronic devices.

This work was supported by DARPA and the Air Force Research Laboratories. We thank L. Biedermann, M. Bolen, and D. Zemlyanov for helpful discussions throughout the course of this work.

${ }^{1}$ V. M. Pereira and A. H. Castro Neto, Phys. Rev. Lett. 103, 046801 (2009).

${ }^{2}$ A. J. Van Bommel, J. E. Crombeen, and A. Van Tooren, Surf. Sci. 48, 463 (1975).

${ }^{3}$ I. Forbeaux, J. M. Themlin, and J. M. Debeveret, Phys. Rev. B 58, 16396 (1998).

${ }^{4}$ E. Rollings, G.-H. Gweon, S. Y. Zhou, B. S. Mun, J. L. McChesney, B. S. Hussain, A. V. Fedorov, P. N. First, W. A. de Heer, and A. Lanzara, J. Phys. Chem. Solids 67, 2172 (2006).

${ }^{5}$ J. Hass, W. A. de Heer, and E. H. Conrad, J. Phys.: Condens. Matter 20, 323202 (2008)

${ }^{6}$ R. M. Tromp and J. B. Hannon, Phys. Rev. Lett. 102, 106104 (2009).

${ }^{7}$ U. Starke and C. Riedl, J. Phys.: Condens. Matter 21, 134016 (2009).

${ }^{8}$ M. L. Bolen, S. E. Harrison, L. B. Biedermann, and M. A. Capano, Phys. Rev. B 80, 115433 (2009).

${ }^{9}$ T. Ohta, F. El Gabaly, A. Bostwick, J. L. McChesney, K. V. Emtsev, A. K. Schmid, T. Seyller, K. Horn, and E. Rotenberg, New J. Phys. 10, 023034 (2008).

${ }^{10}$ H. Hibino, H. Kageshima, F. Maeda, M. Nagase, Y. Kobayashi, and H. Yamaguchi, Phys. Rev. B 77, 075413 (2008).

${ }^{11}$ J. A. Robinson, M. Wetherington, J. L. Tedesco, P. M. Campbell, X. Weng, J. Stitt, M. A. Fanton, E. Frantz, D. Snyder, B. L. VanMil, G. G. Jernigan, R. L. Myers-Ward, C. R. Eddy, Jr., and D. K. Gaskill, Nano Lett. 9, 2873 (2009).

${ }^{12}$ K. I. Bolotin, K. J. Sikes, Z. Jiang, M. Klima, G. Fudenberg, J. Hone, P. Kim, and H. L. Stormer, Solid State Commun. 146, 351 (2008).
${ }^{13}$ J. L. Tedesco, B. L. VanMil, R. L. Meyers-Ward, J. M. McCrate, S. A. Kitt, P. M. Campbell, G. G. Jernigan, J. C. Culbertson, C. R. Eddy, Jr., and D. K. Gaskill, Appl. Phys. Lett. 95, 122102 (2009).

${ }^{14}$ T. Shen, J. J. Gu, M. Xu, Y. Q. Wu, M. L. Bolen, M. A. Capano, L. W. Enel, and P. D. Ye, Appl. Phys. Lett. 95, 172105 (2009).

${ }^{15}$ L. Biedermann, M. L. Bolen, M. A. Capano, D. Zemlyanov, and R. Reifenberger, Phys. Rev. B 79, 125411 (2009).

${ }^{16}$ F. Varchon, P. Mallet, L. Magaud, and J. Y. Veuillen, Phys. Rev. B 77, 165415 (2008).

${ }^{17}$ Three $8 \times 8 \mathrm{~mm}^{2}$ samples were prepared by annealing nominally on-axis, semi-insulating $4 \mathrm{H}-\mathrm{SiC}$ in an Epigress VP508 chemical vapor deposition reactor. The starting wafers were etched at $1500{ }^{\circ} \mathrm{C}$ for $10 \mathrm{~min}$. in hydrogen at 150 mbar. After etching, the growth cell was pumped to a pressure of $\sim 5 \times 10^{-6}$ mbar, the samples were heated to $1100{ }^{\circ} \mathrm{C}$ under high vacuum for $3 \mathrm{~min}$. and then ramped to specific growth temperatures at a rate of $10{ }^{\circ} \mathrm{C} / \mathrm{min}$. All samples were held at temperature for $10 \mathrm{~min}$. and allowed to cool under vacuum.

${ }^{18}$ Prior $\mathrm{x}$-ray photoelectron spectroscopy studies indicate the thickness of the C-layer formed at $1475{ }^{\circ} \mathrm{C}$ is $1.8 \pm 0.1 \mathrm{~nm}$ thick (approximately five layers of graphene) while growth at $1500{ }^{\circ} \mathrm{C}$ produced FLG with a thickness of $2.4 \pm 0.2 \mathrm{~nm}$ (approximately seven layers of graphene). See Ref. 15 .

${ }^{19}$ The STM studies were performed under dry nitrogen conditions using a cut Pt/Ir tip. Each sample was imaged for a period of approximately three or more days, taking $\sim 50$ images per day. A Nanotec Electronica STM was used. The images were analyzed using WSxM software, version 4, develop 13.

${ }^{20}$ I. Horcas, R. Fernandez, J. M. Gomez-Rodriguez, J. Colchero, J. GomezHerrero, and A. M. Baro, Rev. Sci. Instrum. 78, 013705 (2007).

${ }^{21}$ I. A. Nyapshaev, I. V. Makarenko, A. N. Titkov, A. V. Tyurnina, and A. N. Obraztsov, Phys. Solid State 51, 1054 (2009).

${ }^{22}$ The striations are often not visible in dynamic AFM (dAFM) studies due to the inherent lower resolution of dAFM.

${ }^{23}$ H. Hiura, T. W. Ebbesen, J. Fujita, K. Tanigaki, and T. Takada, Nature (London) 367, 148 (1994)

${ }^{24}$ E. M. Kramer and T. A. Witten, Phys. Rev. Lett. 78, 1303 (1997).

${ }^{25}$ The height constraint can be as simple as a rigid ceiling that restricts the ridge height to a fixed value. When a growing ridge continues to press against the ceiling, the ridge is further deformed into parallel ridges of constant height. These parallel ridges can have the appearance of striations that run parallel to the entire length of the original (unconstrained) ridge.

${ }^{26}$ E. Cerda, K. Ravi-Chandar, and L. Mahadevan Nature (London) 419, 579 (2002).

${ }^{27}$ J. C. Géminard, R. Bernal, and F. Melo, Eur. Phys. J. E 15, 117 (2004).

${ }^{28}$ A. F. Lobkovsky, S. Gentges, H. Li, D. Morse, and T. A. Witten, Science 270, 1482 (1995).

${ }^{29}$ A. F. Lobkovsky and T. A. Witten, Phys. Rev. E 55, 1577 (1997).

${ }^{30}$ S. Chaïeb and F. Melo, J. Mech. Phys. Solids 48, 565 (2000).

${ }^{31}$ G. A. Vliegenthart and G. Gompper, Nature Mater. 5, 216 (2006).

${ }^{32}$ M. Das, A. Vaziri, A. Kudrolli, and L. Mahadevan, Phys. Rev. Lett. 98, 014301 (2007).

${ }^{33}$ L. Pocivavsek, R. Dellsy, A. Kern, S. Johnson, B. Lin, K. Y. C. Lee, and E. Cerda, Science 320, 912 (2008).

${ }^{34}$ M. Kuwabara, D. R. Clarke, and D. A. Smith, Appl. Phys. Lett. 56, 2396 (1990).

${ }^{35}$ W. T. Pong and C. Durkan, Jpn. J. Appl. Phys., Part 1 44, 5365 (2005).

${ }^{36}$ W. T. Pong and C. Durkan, J. Phys. D 38, R329 (2005).

${ }^{37}$ It is possible that the 1D periodicities could result from an elongation of the graphene unit cell, in which case the observed periodicity would be specified by $q d=(q-1) d^{\prime}=P ; q=$ integer. Since $4.5 \mathrm{~nm}<P<5.6 \mathrm{~nm}$, it follows that $18<q<23$. Such an argument would require $d^{\prime} / d \sim 1.05$ between adjacent graphene layers. 